\title{
Lower education predicts poor response to dietary intervention in pregnancy, regardless of neighbourhood affluence: secondary analysis from the ROLO randomised control trial
}

\author{
Eileen C O'Brien, Goiuri Alberdi, Aisling A Geraghty and Fionnuala M McAuliffe* \\ UCD Perinatal Research Centre, Obstetrics \& Gynaecology, UCD School of Medicine, University College Dublin, \\ National Maternity Hospital, Dublin, Republic of Ireland
}

Submitted 9 February 2017: Final revision received 29 May 2017: Accepted 20 June 2017: First published online 15 August 2017

\begin{abstract}
Objective: To determine if response to a low glycaemic index (GI) dietary intervention, measured by changes in dietary intake and gestational weight gain, differed across women of varying socio-economic status (SES).

Design: Secondary data analysis of the ROLO randomised control trial. The intervention consisted of a two-hour low-GI dietary education session in early pregnancy. Change in GI was measured using $3 \mathrm{~d}$ food diaries pre- and post-intervention. Gestational weight gain was categorised as per the 2009 Institute of Medicine guidelines. SES was measured using education and neighbourhood deprivation.

Setting: The National Maternity Hospital, Dublin, Ireland.

Subjects: Women ( $n$ 625) recruited to the ROLO randomised control trial.

Results: The intervention significantly reduced GI and excess gestational weight gain (EGWG) among women with third level education residing in both disadvantaged (GI, mean (SD), intervention $v$. control: -3.30 (5.15) $v$. -0.32 (4.22), $P=0.024$; EGWG, $n$ (\%), intervention v. control: 7 (33.6) v. 22 (67.9); $P=0.022)$ and advantaged areas (GI: -1.13 (3.88) v. $0.06(3.75), P=0.020$; EGWG: $41(34.1) v .58(52 \cdot 6) ; P=0.006)$. Neither GI nor gestational weight gain differed between the intervention and control group among women with less than third level education, regardless of neighbourhood deprivation.

Conclusions: A single dietary education session was not effective in reducing GI or gestational weight gain among less educated women. Multifaceted, appropriate and practical approaches are required in pregnancy interventions to improve pregnancy outcomes for less educated women.
\end{abstract}

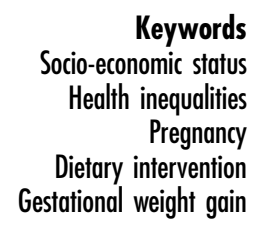

Low socio-economic status (SES) is a determining factor of poor maternal diet ${ }^{(1-4)}$, lifestyle behaviours ${ }^{(5-7)}$ and pregnancy outcomes ${ }^{(8,9)}$. However, the response of women of low SES to dietary interventions in pregnancy is largely unknown. In terms of health, SES is a measure of an individual's access to the basic resources required to achieve good health, and can be estimated by income, occupation, education and neighbourhood $^{(10)}$. The reasons for poorer health and health-related behaviours among disadvantaged groups of the population are manifold, including unemployment, inferior housing conditions, discrimination ${ }^{(11)}$, greater anxiety and depression ${ }^{(12)}$, and barriers to healthy lifestyle choices such as limited food budget, lack of cooking skills and poor nutrition knowledge ${ }^{(13)}$.

Since poor health and health-related behaviours are more prevalent among disadvantaged groups of the population, it would seem prudent that these individuals are provided with resources and support to allow them equal opportunities to influence their own health. Governments and state bodies aim to address this through public health policies and initiatives such as food subsidy programmes, dietary interventions, additional community funding and family support services ${ }^{(14,15)}$. The International Weight Management in Pregnancy (i-WIP) collaboration recommends that public health measures are taken to promote lifestyle education and behaviour change before, during and after pregnancy among women of low SES ${ }^{(16)}$. However, if policies that meet the needs of disadvantaged groups are to be successful, research specific to these individuals must be carried out that will inform governments and public health bodies. Currently, research and intervention studies, through their design, analysis and dissemination, are not filling the knowledge gaps which exist in relation to initiatives among individuals of low SES. 
Notably, there is a paucity of data on the response of low-SES individuals to interventions, as the majority of clinical trials and observational studies do not analyse the outcomes of interest stratified by SES, and individuals of low SES are not represented in equal numbers ${ }^{(17-19)}$. The lack of consideration of the differences in outcomes across socio-economic groups makes it difficult for readers, health-care professionals and policy makers to disentangle the types of interventions that have the greatest impact among those of lower $\mathrm{SES}^{(17)}$.

For policies to be successful in changing health behaviours, multifaceted approaches with both upstream interventions (those that influence policy) and downstream interventions (those that influence individual-level behaviours) are required in tandem ${ }^{(20)}$. However, the majority of research studies carried out in the context of dietary interventions in pregnancy are designed as single downstream interventions ${ }^{(21-24)}$. More information is required to understand if these interventions are successful in changing behaviours among low-SES women, and strategies to improve response.

The ROLO study (Randomised cOntrol trial of a LOw glycaemic index diet in pregnancy to prevent macrosomia) consisted of low glycaemic index (GI) dietary education session, delivered in small groups, to pregnant women ${ }^{(21)}$. Since nutrition knowledge is a mediator between SES and diet quality ${ }^{(1)}$, a dietary group education session may be appropriate for women of low SES. While the low-GI dietary intervention was successful in reducing dietary GI and gestational weight gain ${ }^{(21)}$, differences in these outcomes across socio-economic groups (education level and neighbourhood deprivation) have not been examined to date. We aimed to determine if response to a low-GI dietary intervention, measured by changes in diet and excess gestational weight gain, differed across women of high/low educational attainment and neighbourhood deprivation.

\section{Participants and methods}

\section{Study design}

The present study was a secondary data analysis of 625 pregnant women originally recruited as part of the ROLO study between 2007 and 2011, at the National Maternity Hospital, Dublin, Ireland. Detailed methodology and results of the ROLO study have been previously published $^{(21,25)}$. In brief, 800 secundigravida women who had previously given birth to a macrosomic infant (>4000 g) were randomised to receive either low-GI dietary advice from the research dietitian or standard usual care (no dietary advice), with the aim to reduce recurrence of macrosomia ${ }^{(21)}$. Although the primary outcome of birth weight did not differ significantly between the intervention and control arms, the secondary outcomes, gestational weight gain, dietary GI and glucose intolerance, were reduced significantly in the intervention $\operatorname{arm}^{(21,25)}$.

\section{Patient selection}

Participants were recruited at the first antenatal hospital visit between 10 and 18 weeks' gestation. Women were included in the study if they were aged 18 years or above, had previously delivered one infant weighing $\geq 4000 \mathrm{~g}$ and had good understanding of the English language. They were excluded if they were pregnant with twins/triplets, had previous or current gestational diabetes, or had an underlying medical condition requiring medication.

\section{Intervention}

Participants were randomised into the intervention or control arm using computer-generated allocations. Two weeks post-randomisation, women in the intervention group attended one two-hour group education session with the research dietitian, in groups of two to six participants. They received low-GI dietary advice and were encouraged to choose low-GI alternatives to high-GI carbohydrate foods. They were also advised on general healthy eating recommendations for pregnancy. Information leaflets were provided to participants that included low-GI recipes, lists of low-GI foods, fact sheets and tips. The research dietitian met with the intervention participants at 28 and 34 weeks' gestation for brief reinforcement of the low-GI diet and to answer any questions about the diet.

\section{Anthropometry}

At the first antenatal consultation (randomisation), participants were weighed in light clothing using a SECA weighing scale (SECA GmbH \& Co. KG, Germany) to the nearest $0 \cdot 1 \mathrm{~kg}$ and height was measured without shoes to the nearest $0 \cdot 1 \mathrm{~cm}$ using a wall-mounted stadiometer. BMI $\left(\mathrm{kg} / \mathrm{m}^{2}\right)$ was calculated. Mid-upper arm circumference was measured at the first antenatal consultation using a SECA non-stretch measuring tape.

\section{Gestational weight gain}

Maternal weight was recorded and gestational weight gain calculated at multiple time points throughout pregnancy: 24 , 28, 34, 38, 40 and 41 weeks' gestation. A final weight measurement at 38, 40 or 41 weeks' gestation was available for 343 participants. The measurement taken at the latest gestation was considered the final weight. The last weight measurement was taken at 34 weeks' gestation for an additional 178 participants. For these women, weight at 38 weeks' gestation was imputed (see methods for multiple imputation below). A further 104 participants did not have a weight measurement at 34, 38, 40 or 41 weeks' gestation. These women were excluded from the analysis. The maternal characteristics of the women included and excluded from the gestational weight gain analysis are included in the online supplementary material, Supplemental Table 1.

Total gestational weight gain was calculated by subtracting the measured weight at the first antenatal visit from the final weight. Gestational weight gain per week was calculated by dividing total weight gained by the 
number of weeks of gestation (measured final weight) or by 38 (imputed final weight). The 2009 Institute of Medicine (IOM) guidelines for total gestational weight gain ${ }^{(26)}$ and BMI at the first antenatal visit were used to categorise gestational weight gain as inadequate, adequate or excessive. For analysis, we dichotomised gestational weight gain as 'exceeded IOM guidelines' and 'did not exceed IOM guidelines'.

\section{Nutrient intakes}

Food and beverages consumed over three consecutive days were recorded during the first trimester (prior to intervention education session) and during both the second and third trimesters. Dietary data from food diaries were entered into the dietary analysis software NetWISP version 3.0 (Tinuviel Software, Llanfechell, UK). Change in nutrient intake from pre- and post-intervention was calculated by subtracting the baseline nutrient intake from the mean of the second- and third-trimester intakes.

\section{Lifestyle characteristics}

Emotional well-being was examined using the WHO-5 Wellbeing Index ${ }^{(27)}$. The questionnaire contains five statements that describe positive moods. Each statement is rated on 6-point Likert scales, based on mood during the previous two weeks. Responses were standardised to a percentage score, with $100 \%$ indicating maximal wellbeing ${ }^{(28)}$. Smoking status, age and gestational age at the first visit were also recorded.

\section{Socio-economic status}

Educational attainment was self-reported. Participants selected one of six categories: complete third level (higherlevel degree); some third level (certificate/diploma); complete secondary level; some secondary level; primary education only; or no education. The Pobal Haase \& Pratschke Deprivation Index (HP Index) was used to assign deprivation indices at street level, or small area ${ }^{(29)}$. The HP Index is based on data from the 2011 Census of Population in Ireland, with information on three measurements of affluence/disadvantage incorporated: demographic profile; social class composition; and labour market situation (Fig. 1). The HP Index is normally distributed, and the mean is set as 0 . Therefore, it can be determined if a small area is disadvantaged (negative) or advantaged (positive). HP Index scores can be categorised from 'extreme affluence' to 'extreme deprivation'. The participant's street address was entered into the online interactive mapping tool (available at http://maps.pobal.ie/\#/Map) and the score was recorded. Due to small numbers in the extremely affluent group $(n 2)$, these women were categorised as 'very affluent'.

We created a deprivation-education variable that categorised participants into one of four groups based on advantaged or disadvantaged neighbourhood deprivation and education level above or below third level (university). Categories were as follows: 'disadvantaged and $<3$ rd level'; 'disadvantaged and $\geq 3$ rd level'; 'advantaged and $<3$ rd level'; and 'advantaged and $\geq 3$ rd level'.

\section{Compliance and acceptability}

At 34 weeks' gestation, participants in the intervention group completed a questionnaire to access compliance and acceptability of the low-GI dietary intervention. Participants indicated level of compliance with the intervention on a 5-point Likert scale. The acceptability questionnaire consisted of six questions, also 5-point Likert scales, exploring whether the participant found the dietary intervention easy to follow, enjoyable, economical, provided her with enough energy and variety, and if her family were satisfied with changes.

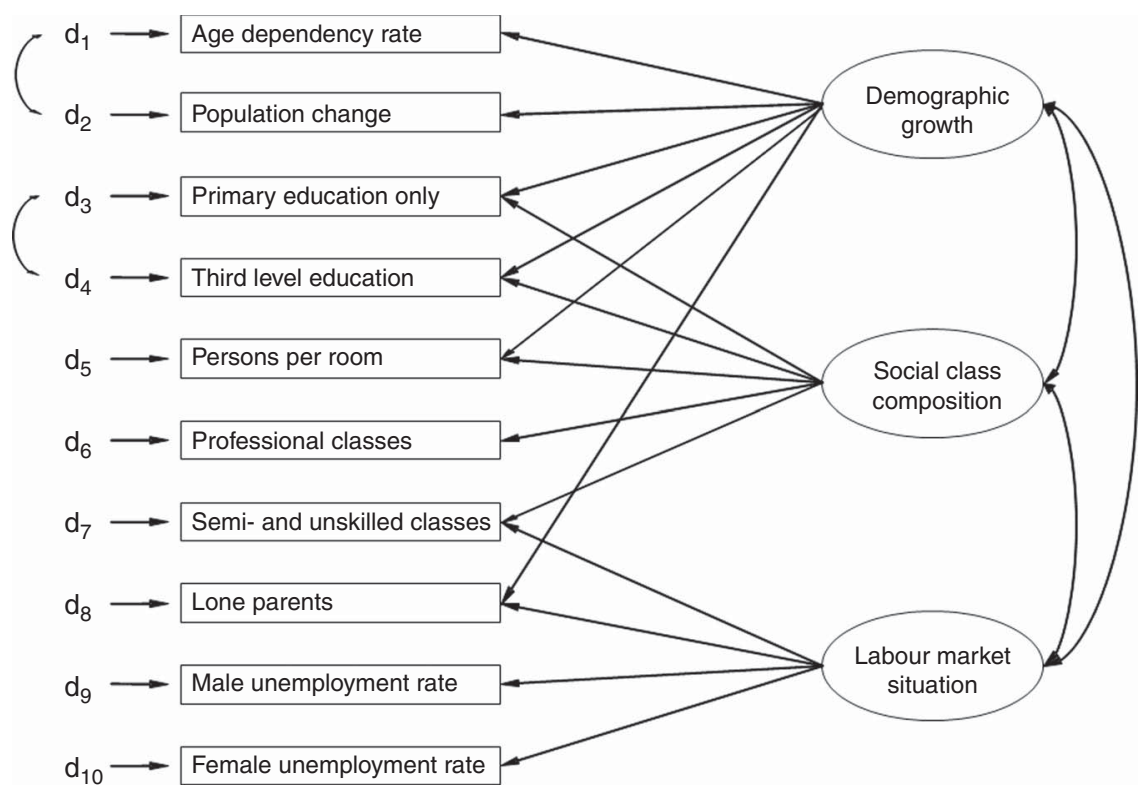

Fig. 1 Basic model of the Pobal Haase \& Pratschke Deprivation Index (HP Index) ${ }^{(29)}$ 


\section{Multiple imputation}

We used multiple imputation to derive missing values for 38-week weight, using the weights at booking, weeks 34, 40 and 41, as well as smoking status and age. Five imputations were performed, convergence verified and the imputed values were reasonably close to observed values. Calculation of the weight gain was then performed on the raw imputed values as described above, and pooled analyses performed over all imputed data sets.

\section{Statistical analysis}

Data were assessed for normality using the KolmogorovSmirnov test and visual inspection of histograms. One-way ANOVA with post hoc Tukey's tests were used to explore maternal characteristics across deprivation and education categories. Independent-sample $t$ tests explored differences between maternal characteristics in the intervention and control groups, within deprivation and education categories. Excess gestational weight gain was analysed using binary logistic regression. Changes in nutrient intake from pre-intervention to post-intervention were analysed using independent-sample $t$ tests. Compliance and acceptability of the intervention were analysed using one-way ANOVA with post hoc Tukey's test and $\chi^{2}$ tests. Well-being was compared using independent-sample $t$ tests. All statistical analyses were performed using the statistical software package IBM SPSS Statistics for Windows, version 20.0. Results were considered statistically significant if $P<0 \cdot 05$.

\section{Results}

\section{Maternal characteristics}

The demographic details for the total study population ( $n$ 625) are displayed in Table 1. Mean early-pregnancy BMI was $26.6 \mathrm{~kg} / \mathrm{m}^{2}$. Three (0.5\%) participants were underweight, $270(43.2 \%)$ were normal weight, 238 (38.1\%) were classified as overweight and 114 (18.2\%) were obese. Half of the population ( $n$ 299, 47.8\%) received the low-GI dietary intervention. The comparison of maternal characteristics across deprivation-education categories is displayed in Table 2. Women in the 'disadvantaged and <3rd level' category were significantly younger and had the lowest WHO-5 Wellbeing scores compared with women in other deprivation-education categories. This group of women also had higher earlypregnancy BMI and mid-upper arm circumference than those in the 'advantaged and $\geq 3$ rd level' category. There were few differences in maternal characteristics between the intervention and control groups across each of the deprivation-education categories (see online supplementary material, Supplemental Table 2). WHO-5 Wellbeing score was higher in the intervention participants of the 'disadvantaged and $\geq 3$ rd level' and 'advantaged and $<3$ rd level' categories.
Table 1 Maternal characteristics of women ( $N$ 625) recruited to the ROLO randomised control trial, Dublin, Ireland, 2007-2011

\begin{tabular}{|c|c|c|c|}
\hline Maternal characteristic & $N$ & Mean & SD \\
\hline Age (years) & 625 & 32.64 & 4.04 \\
\hline Gestation at delivery (d) & 625 & 281.92 & 8.00 \\
\hline Weight at 1st antenatal visit (kg) & 625 & 73.30 & $13 \cdot 76$ \\
\hline BMI at 1 st antenatal visit $\left(\mathrm{kg} / \mathrm{m}^{2}\right)$ & 625 & 26.57 & 4.77 \\
\hline MUAC at 1st antenatal visit (cm) & 608 & 29.40 & 3.41 \\
\hline Weight at final visit $(\mathrm{kg})$ & 521 & 86.91 & 14.01 \\
\hline Total GWG (kg) & 521 & $13 \cdot 13$ & 4.44 \\
\hline GWG per week (kg/week) & 521 & 0.34 & 0.12 \\
\hline HP Index score & 625 & $5 \cdot 61$ & $10 \cdot 19$ \\
\hline \multirow[t]{2}{*}{ WHO-5 Wellbeing score (\%) } & 615 & $58 \cdot 17$ & $15 \cdot 41$ \\
\hline & $N$ & $n$ & $\%$ \\
\hline Smoking, yes & 625 & 23 & 3.68 \\
\hline Ethnicity & 625 & & \\
\hline White Irish & & 560 & 89.60 \\
\hline White Other & & 51 & $8 \cdot 16$ \\
\hline Other & & 14 & $2 \cdot 24$ \\
\hline Education level & 625 & & \\
\hline Completed third level (higher-level degree) & & 348 & $55 \cdot 68$ \\
\hline Some third level (certificate/diploma) & & 140 & $22 \cdot 40$ \\
\hline Completed secondary level & & 104 & $16 \cdot 64$ \\
\hline Some secondary level & & 33 & $5 \cdot 28$ \\
\hline HP Index & 625 & & \\
\hline Very affluent & & 39 & $6 \cdot 24$ \\
\hline Affluent & & 185 & 29.60 \\
\hline Marginally above average & & 247 & $39 \cdot 52$ \\
\hline Marginally below average & & 99 & $15 \cdot 84$ \\
\hline Disadvantaged & & 46 & $7 \cdot 36$ \\
\hline Very disadvantaged & & 9 & 1.44 \\
\hline Deprivation-education category & 625 & & \\
\hline Advantaged and $\geq 3$ rd level & & 283 & $45 \cdot 28$ \\
\hline Advantaged and $<3$ rd level & & 188 & 30.08 \\
\hline Disadvantaged and $\geq 3$ rd level & & 65 & $10 \cdot 40$ \\
\hline Disadvantaged and $<3$ rd level & & 89 & $14 \cdot 24$ \\
\hline
\end{tabular}

ROLO, Randomised cOntrol trial of a LOw glycaemic index diet in pregnancy to prevent macrosomia; MUAC, mid-upper arm circumference; GWG; gestational weight gain; HP Index, Pobal Haase \& Pratschke Deprivation Index.

\section{Excess gestational weight gain}

Data on deprivation-education category and excess gestational weight gain were available for 521 participants. Within the 'disadvantaged and $\geq 3$ rd level' and 'advantaged and $\geq 3$ rd level' categories, those in the intervention group were significantly less likely to gain excess weight compared with those in the control group (Table 3). There was no significant difference in excess gestational weight gain between participants in the intervention and control groups in the 'disadvantaged and $<3$ rd level' and 'advantaged and <3rd level' categories. Furthermore, total weight gain and weight gain per week were significantly lower among women in the intervention group compared with the control group in the 'disadvantaged and $\geq 3$ rd level' and 'advantaged and $\geq 3$ rd level' categories (see online supplementary material, Supplemental Table 2).

Women excluded from the gestational weight gain analysis ( $n$ 104) had significantly lower weight, BMI and mid-upper arm circumference at the randomisation visit, but their neighbourhood deprivation score was not significantly different from that of the women included (see online supplementary material, Supplemental Table 1). 
Table 2 Maternal characteristics according to deprivation-education category of women $(N 625)$ recruited to the ROLO randomised control trial, Dublin, Ireland, 2007-2011

\begin{tabular}{|c|c|c|c|c|c|c|c|c|c|}
\hline \multirow[b]{2}{*}{ Maternal characteristic } & \multicolumn{2}{|c|}{$\begin{array}{c}\text { Disadvantaged } \\
\text { and }<3 \text { rd level } \\
\text { (a) }\end{array}$} & \multicolumn{2}{|c|}{$\begin{array}{l}\text { Disadvantaged } \\
\text { and } \geq 3 \text { rd level } \\
\text { (b) }\end{array}$} & \multicolumn{2}{|c|}{$\begin{array}{c}\text { Advantaged } \\
\text { and }<3 \text { rd level } \\
\text { (c) }\end{array}$} & \multicolumn{2}{|c|}{$\begin{array}{c}\text { Advantaged } \\
\text { and } \geq 3 \text { rd level } \\
\text { (d) }\end{array}$} & \multirow[b]{2}{*}{$P^{*}$} \\
\hline & Mean & SD & Mean & SD & Mean & SD & Mean & SD & \\
\hline Age (years) & $30 \cdot 44^{\mathrm{b}, \mathrm{c}, \mathrm{d}}$ & $4 \cdot 77$ & $32.44^{\mathrm{a}}$ & 4.07 & $32 \cdot 03^{a, d}$ & 4.03 & $33 \cdot 77^{\mathrm{a}, \mathrm{c}}$ & 3.38 & $<0.001$ \\
\hline Gestation at delivery (d) & 282.49 & $7 \cdot 70$ & $282 \cdot 32$ & 8.41 & 281.45 & 7.95 & 281.96 & 8.05 & 0.733 \\
\hline Weight at 1 st antenatal visit $(\mathrm{kg})$ & $76.39^{d}$ & $14 \cdot 30$ & 74.02 & $13 \cdot 16$ & 74.25 & 14.54 & $71.53^{\mathrm{a}}$ & 12.98 & 0.016 \\
\hline BMI at 1 st antenatal visit $\left(\mathrm{kg} / \mathrm{m}^{2}\right)$ & $28.09^{d}$ & 4.95 & $26 \cdot 84$ & 4.66 & $27 \cdot 24^{d}$ & 5.03 & $25 \cdot 59^{\mathrm{a}, \mathrm{c}}$ & 4.35 & $<0.001$ \\
\hline MUAC at 1 st antenatal visit $(\mathrm{cm})$ & $30.02^{d}$ & $3 \cdot 10$ & 29.80 & 3.31 & 29.64 & 3.87 & $28.92^{\mathrm{a}}$ & $3 \cdot 13$ & 0.018 \\
\hline Weight at final visit $(\mathrm{kg})$ & $89 \cdot 21$ & $12 \cdot 75$ & $90 \cdot 08$ & 12.59 & 88.06 & $15 \cdot 47$ & 85.53 & $13 \cdot 16$ & 0.153 \\
\hline Total GWG (kg) & 13.59 & 4.74 & $13 \cdot 40$ & $4 \cdot 80$ & $12 \cdot 75$ & 4.82 & $13 \cdot 53$ & 4.41 & 0.575 \\
\hline GWG per week (kg/week) & 0.34 & 0.12 & 0.34 & 0.12 & 0.32 & 0.12 & 0.34 & 0.11 & 0.566 \\
\hline WHO-5 Wellbeing score (\%) & $52 \cdot 40^{\mathrm{b}, \mathrm{c}, \mathrm{d}}$ & $16 \cdot 80$ & $61 \cdot 60^{\mathrm{a}}$ & $14 \cdot 60$ & $58 \cdot 20^{\mathrm{a}}$ & $15 \cdot 20$ & $59 \cdot 20^{\mathrm{a}}$ & 14.90 & 0.001 \\
\hline
\end{tabular}

ROLO, Randomised cOntrol trial of a LOw glycaemic index diet in pregnancy to prevent macrosomia; MUAC, mid-upper arm circumference; GWG; gestational weight gain.

$a, b, c, d$ Mean value was significantly different from that in the deprivation-education category denoted by the letter.

${ }^{\star} P$ value is for one-way ANOVA with post hoc Tukey's test.

Table 3 Intervention and risk of excessive gestational weight gain according to deprivation-education category of women ( $N 521)$ recruited to the ROLO randomised control trial, Dublin, Ireland, 2007-2011

\begin{tabular}{|c|c|c|c|c|c|}
\hline \multirow[b]{2}{*}{ Deprivation-education category } & \multirow[b]{2}{*}{ Study group } & \multicolumn{2}{|c|}{ Exceeded IOM guideline ${ }^{(26)}$} & \multirow[b]{2}{*}{ OR } & \multirow[b]{2}{*}{$P$} \\
\hline & & $n$ & $\%$ & & \\
\hline \multirow[t]{2}{*}{ Disadvantaged and $<3$ rd level } & Intervention & 27 & $55 \cdot 51$ & \multirow[t]{2}{*}{0.924} & \multirow[t]{2}{*}{0.882} \\
\hline & Control & 18 & 57.50 & & \\
\hline \multirow[t]{2}{*}{ Disadvantaged and $\geq 3$ rd level } & Intervention & 7 & 33.64 & \multirow[t]{2}{*}{0.239} & \multirow[t]{2}{*}{0.022} \\
\hline & Control & 22 & 67.88 & & \\
\hline \multirow[t]{2}{*}{ Advantaged and $<3$ rd level } & Intervention & 29 & $45 \cdot 63$ & \multirow[t]{2}{*}{0.769} & \multirow[t]{2}{*}{0.465} \\
\hline & Control & 48 & $52 \cdot 17$ & & \\
\hline \multirow[t]{2}{*}{ Advantaged and $\geq 3$ rd level } & Intervention & 41 & $34 \cdot 12$ & \multirow[t]{2}{*}{0.467} & \multirow[t]{2}{*}{0.006} \\
\hline & Control & 58 & 52.55 & & \\
\hline
\end{tabular}

ROLO, Randomised cOntrol trial of a LOw glycaemic index diet in pregnancy to prevent macrosomia; IOM, Institute of Medicine. OR derived from binary logistic regression.

\section{Nutrient intakes}

The mean changes in nutrient intakes from preintervention to post-intervention are shown in Table 4. The intervention reduced GI significantly in both the 'advantaged and $\geq 3$ rd level' and 'disadvantaged and $\geq 3$ rd level' categories. The reduction in GI in the intervention groups of the other deprivation-education categories did not reach statistical significance. Additionally, the intervention significantly reduced glycaemic load, total carbohydrate and total sugars within the 'advantaged and $\geq 3$ rd level' category. It also reduced glycaemic load in the 'advantaged and <3rd level' category. The intervention did not significantly change any of the nutrient intakes from pre-intervention to post-intervention in the 'disadvantaged and $<3$ rd level' category.

\section{WHO-5 Wellbeing score}

Compared with participants in the control arm, the WHO-5 Wellbeing score was significantly lower in the intervention arm within the 'disadvantaged and $\geq 3$ rd level' category (intervention: 56.7 (SD 15.3) $v$. control: 64.8 (SD 13.3), $P=0.028$ ) and 'advantaged and <3rd level' category (intervention: 54.8 (SD 16.0) $v$. control: $60 \cdot 6$ (SD 14.1), $P=0.010)$. There was no difference between the intervention and control arms of the study within the 'disadvantaged and <3rd level' (intervention: $50 \cdot 6$ (SD 18.2) $v$. control: 55.5 (SD 13.9), $P=0.148$ ) or 'advantaged and $\geq 3 \mathrm{rd}$ level' (intervention: 58.8 (sD 13.3) v. control: $59 \cdot 5$ (sD 16.3), $P=0.692)$ category.

\section{Compliance and acceptability}

Of the 183 participants in the intervention arm who completed the compliance and acceptability questionnaire, $80.3 \%$ reported following the prescribed diet always or most of the time. There was no significant difference in compliance between the categories of deprivation and education score; 'always' or 'mostly' was reported by $79 \cdot 3 \%$ of 'disadvantaged and <3rd level'; $89.5 \%$ of 'disadvantaged and $\geq 3$ rd level'; $74.1 \%$ of 'advantaged and <3rd level'; and $82.7 \%$ of 'advantaged and $\geq 3$ rd level' $(P=0 \cdot 761)$. Most participants $(68 \cdot 8 \%)$ found the recommended diet easy to follow, with no differences observed between SES groups $(P=0 \cdot 183)$. Within the total group, $65.6 \%$ of participants reported that they 
enjoyed the dietary changes made, which was similar between the categories of deprivation and education score $(P=0.666)$. The majority, $81.8 \%$, agreed that the changes they made did not increase their weekly grocery bill, with no differences observed between SES groups $(P=0.673)$. Seventy-nine per cent felt they had enough energy while on the diet, which was similar across the groups $(P=0.191)$. Among the total group, $83.1 \%$ agreed that they enjoyed a wide variety of foods in their eating plan, with no differences observed between SES groups $(P=0 \cdot 144)$. Overall, $71.4 \%$ agreed that their family was happy with the changes made to their diet. This was significantly lower in the 'disadvantaged and $\geq 3$ rd level' category, within which $40.0 \%$ agreed that their family was happy $(P=0 \cdot 040)$.

\section{Discussion}

The main finding from the present study is that an intervention which consisted of a single dietary group education session, with brief reinforcement, significantly reduced excess gestational weight gain and improved dietary GI only among women with third level education, regardless of neighbourhood deprivation. Neither gestational weight gain nor GI differed between the intervention and control groups among women who had not attended third level education, residing in both advantaged and disadvantaged neighbourhoods. Since the dietary education session was not effective among less educated women, it would seem prudent that tailored approaches are required to increase the effectiveness of interventions among women of lower educational attainment.

Huynh et al. examined both education and neighbourhood disadvantage in relation to excess gestational weight gain. High educational attainment was protective against excess gestational weight gain and residing in a disadvantaged neighbourhood increased the risk ${ }^{(30)}$. Unlike our findings, women with a high level of education were at an increased risk of excess weight gain if they resided in a disadvantaged neighbourhood, suggesting that the protective effect of high maternal education was attenuated by the negative social environments ${ }^{(30)}$. Many studies have reported that low educational attainment is associated with excess gestational weight gain ${ }^{(31-33)}$, but this is not conclusive $^{(34-36)}$. A large, multicentre cohort study found no association between socio-economic index and excess gestational weight gain $^{(37)}$. The UPBEAT randomised control trial consisted of a low-GI diet among obese pregnant women residing in areas of high socio-economic deprivation in the $\mathrm{UK}^{(38)}$. The intervention was successful in reducing total gestational weight gain among these women of low $\operatorname{SES}^{(38)}$. Although we did not find a reduction in excess gestational weight gain in the most disadvantaged group, the UPBEAT study had a more intense intervention than the ROLO study. Multiple 
support tools and opportunities to meet health trainers in an individual and group context were provided, which may have facilitated behaviour change. Excess gestational weight gain in pregnancy predicts weight retention at 1 year postpartum, and this predisposes women to a BMI in the overweight category 15 years post-pregnancy ${ }^{(39)}$. This is particularly pertinent for low-SES women, as we found that women in the most disadvantaged group began pregnancy with the highest BMI and MUAC.

In terms of dietary changes related to the ROLO low-GI intervention, the most disadvantaged women did not change their diet from pre- to post-intervention, but the most advantaged women significantly reduced GI, glycaemic load, total carbohydrate and total sugars. A single education session with brief reinforcement appeared to be insufficient to change dietary behaviour among the most disadvantaged women. The multifaceted approach of the UPBEAT trial significantly reduced GI and glycaemic load among a mostly disadvantaged population ${ }^{(38)}$. The LIMIT trial in overweight and obese pregnant women found that a healthy eating intervention improved diet quality, but not GI or glycaemic load, although analyses were not stratified by SES and thus cannot be fully compared with our findings ${ }^{(40)}$. Identifying successful interventions for dietary change among those of low SES is important, as these women already have poorer dietary habits ${ }^{(1-3)}$ and nutritional knowledge compared with their high-SES counterparts $^{(1)}$.

\section{Barriers to effective intervention response}

Many women of low SES experience barriers that prevent them from making dietary changes, including lower income, limited food budget, lack of transportation to larger supermarkets, poor cooking skills and family preferences $^{(13,41)}$. These women have stronger beliefs in the influence of chance on health ${ }^{(4)}$, thus they may not prioritise healthy eating. Other barriers include following cravings and difficulty managing time, particularly if the woman has children at home ${ }^{(42)}$. The ROLO dietary intervention seemed to be equally acceptable among the four deprivation-education categories. All groups similarly reported that the intervention was easy to follow, did not increase their weekly grocery bill and was enjoyable, suggesting that, for the majority, the intervention did not increase barriers relating to finances and food preferences. Participants in the 'disadvantaged and $<3$ rd level' category did not report any problems with the intervention compared with their peers; however, since this was the group least likely to make the prescribed changes to their diet, it may be hypothesised that these women did not understand the intervention and did not know what changes were required. Thus, due to lack of changes made in their diet, it is possible that they did not find the intervention difficult. We found that only $40 \%$ of those in the 'disadvantaged and $\geq 3$ rd level' category reported that their families were happy with the changes made to their diet, compared with the average of $71 \%$ across all groups. This could represent a lack of family support.

Those living in disadvantaged areas are likely to experience greater anxiety and depression ${ }^{(12)}$, and low-income women with increased stress are more likely to consume a poor diet ${ }^{(43)}$. In addition, worry about weight gain during pregnancy is positively associated with total gestational weight gain ${ }^{(44)}$. We found that women in the 'disadvantaged and <3rd level' category had the lowest WHO-5 Wellbeing score, which may have been a barrier in making dietary changes. Additionally, the intervention seemed to have a negative impact on well-being score within the 'disadvantaged and $\geq 3$ rd level' and 'advantaged and $<3$ rd level' categories. These women found the dietary changes most stressful to make. Those in the 'disadvantaged and $\geq 3$ rd level' category may have understood the changes required, but did not have the financial, peer or family support required to easily modify their lifestyle.

\section{Tools to facilitate response to interventions}

The principles of health promotion, as set out in the Ottawa Charter for Health Promotion, are building healthy public policy, creating a supportive environment, strengthening community action, developing personal skills and reorienting health services ${ }^{(45)}$. In order for individuals to achieve their greatest health potential, many factors are required, including life skills, information and opportunities to make healthy choices ${ }^{(45)}$. Although engaging with low-SES women may be difficult, as they are less likely to attend antenatal classes ${ }^{(4)}$, it should be a public health priority to provide these women with the relevant tools and information they require to overcome barriers and make healthy choices in a facilitating environment. As researchers, we need to design interventions better to serve the needs of low-SES women.

This raises the question: are different interventions required to target the same outcomes among populations of varying SES? As demonstrated by our findings, the provision of information through an education session is effective only among those of higher educational attainment. Practical tools to enhance life skills, such as growing, cooking and nutrition courses, may help disadvantaged populations to make the healthy choice the easiest choice. Cooking skill interventions among low-SES groups improve confidence in cooking and fruit and vegetable consumption ${ }^{(46)}$, while those involved in community gardening projects also have improved fruit and vegetable intakes ${ }^{(47)}$. Behaviour change techniques, such as barrier identification, goal setting and self-monitoring, may also have some benefit in improving GI, glycaemic load, carbohydrate (percentage of energy) and fibre in pregnant women of low SES ${ }^{(38)}$.

\section{Patient and public involvement in intervention design}

To gain greater understanding of the initiatives and tools that are acceptable to low-SES groups, researchers should seek input from these individuals. Patient and public 
involvement (PPI) is a key component of research and should be utilised particularly in research relating to pregnant women of low-SES groups, who are less likely to engage in health services ${ }^{(48)}$. It encourages research to be carried out with the involvement of society members, rather than for them ${ }^{(49)}$, and strengthens the potential of research to effectively influence practice ${ }^{(50)}$. Participants involved in PPI feel empowered, valued, and develop life skills and knowledge about their condition ${ }^{(51)}$. A positive experience with PPI has also been shown to motivate participants to continue involvement in research ${ }^{(51)}$.

\section{Equality in intervention design}

Researchers and policy makers have a responsibility to create and monitor interventions that provide equal benefit for all members of society, regardless of socio-economic background. Intervention-generated inequalities occur when an intervention is of greater benefit to advantaged (lower-risk) groups than to disadvantaged (higher-risk) groups $^{(52)}$. The ROLO study improved excess gestational weight gain and dietary GI among highly educated women, but not among those with less than third level education. It has been suggested that downstream interventions (which target individual-level behaviours), such as education and media campaigns, increase intervention-generated inequalities $^{(20,52)}$, while upstream interventions (policy approaches), through regulation, increased access or economic incentives, reduce intervention-generated inequalities ${ }^{(20,52)}$. The ROLO study may not have been successful in reaching the most disadvantaged individuals due to its nature as a downstream intervention that provided one education session, at an individual level. Food subsidy programmes are an example of upstream interventions, such as the Healthy
Start programme in the UK, the Special Supplemental Nutrition Programme for Women, Infants, and Children in the USA and the OLO (oufs, lait, orange - eggs, milk, orange) food and supplementation programme in Quebec $^{(53)}$. Food vouchers can increase fruit, vegetable and whole grains consumption ${ }^{(14,54)}$. Home-visiting interventions within disadvantaged communities, such as the NurseFamily Partnership Program in the USA ${ }^{(55)}$ or Preparing for Life in Ireland ${ }^{(56)}$, take a broader view of addressing the health and social needs of low-SES pregnant women. Home visits provide education and support during pregnancy, and have been found to improve pregnancy outcomes ${ }^{(55,56)}$. They also have a positive effect on diet quality, smoking and seeking support services ${ }^{(55)}$.

Within the ROLO study, pregnant women of lower SES and educational attainment may have benefited from multiple, practical downstream approaches to increase knowledge and skills, in addition to upstream, supportive services and economic incentives that would facilitate behaviour change. Figure 2 provides examples of upstream and downstream interventions that could work in tandem to improve gestational weight gain and diet in pregnancy.

\section{Recruitment, analysis and dissemination of interventions}

There is a requirement for researchers to include more disadvantaged women in trials and to stratify analyses by socio-economic groups to allow readers, health-care professionals and policy makers to fully understand the interventions that are most likely to benefit those of lower $\mathrm{SES}^{(17)}$. It is somewhat difficult to compare our findings with the majority of dietary clinical trials and observational

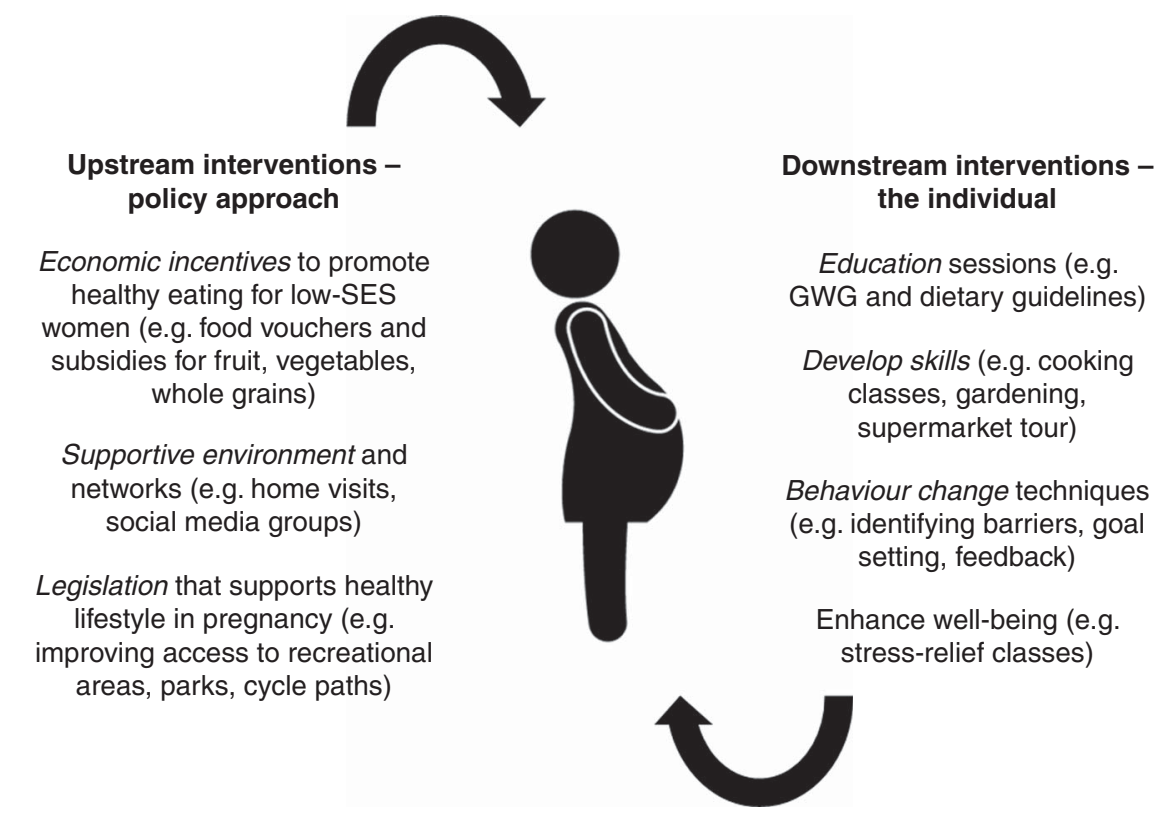

Fig. 2 Potential upstream and downstream interventions to improve excess gestational weight gain and dietary changes in pregnancy (SES, socio-economic status; GWG, gestational weight gain) 
studies among pregnant women, as several studies enrol a greater proportion of high-SES participants and fail to stratify their results by SES. This is not unique to pregnancy research; only 5 and $12 \%$ of the literature on CVD and stroke interventions, respectively, report on intervention effectiveness between socio-economic groups ${ }^{(17,18)}$. Furthermore, less than $10 \%$ of randomised controlled trials published in international medical journals report demographic characteristics relating to SES of their participants ${ }^{(19)}$.

\section{Study strengths and limitations}

The present study has several strengths worthy of consideration. The study design allowed for the measurement of two SES variables: maternal educational attainment and neighbourhood deprivation (HP Index). Combining the two variables to create a deprivation-education variable allowed for categorisation of the most advantaged and disadvantaged groups of individuals within the study population. Additionally, the HP Index is a novel method of determining SES and is unique and specific to the population of Ireland. The study is not without limitations. Nutrient intake data were collected using self-reported food diaries, thus the possibility of error due to memory or social desirability bias must be considered. For those who did not have a final weight recorded, the 38-week gestation weight was imputed. For analysis, the variable 'did not exceed IOM guidelines' included both adequate and inadequate weight gain. Of the 270 participants who did not exceed the IOM guidelines, eighty-six participants gained inadequately. The number of participants in the disadvantaged groups were small, thus the power to detect significant differences between groups may have been reduced. Furthermore, due to multiple testing, some findings may represent false positives and require replication in future studies. If we applied the corrected $P$ value of 0.00135 (due to thirty-seven tests), the only finding to survive correction would be change in glycaemic load from pre-intervention to post-intervention among the participants in the intervention arm within the 'advantaged and $\geq 3$ rd level' category. Lastly, we acknowledge that data on income or occupation would have provided a more complete set of SES variables, but these were not collected.

\section{Conclusions}

Women with third level education, regardless of the neighbourhood in which they lived, were most receptive to the ROLO low-GI dietary intervention. A single, group education session, with brief reinforcement, was not effective in reducing GI and excess gestational weight gain among less educated women. Based on our findings, we recommend the following: (i) researchers should engage in PPI, prior to and during study design, to gain an understanding of participant needs, personal skills required and barriers that prevent lifestyle changes; (ii) create multifaceted, appropriate and practical interventions that combine upstream and downstream approaches, and will engage with and benefit individuals of low SES; (iii) include multiple measures of SES during data collection; and (iv) stratify data analysis by SES to understand the effect of interventions on various population groups. Research is required to drive evidence-based practice, but there remains a paucity of high-quality data on the population of low-SES pregnant women. Thus, there is a requirement for researchers to rethink current strategies and carefully plan meaningful and successful interventions to ultimately reduce the gap in health inequalities.

\section{Acknowledgements}

Acknowledgements: The authors would like to thank the mothers who participated in the study. Financial support: This work was supported by the Health Research Board, Health Research Centre for Health and Diet Research, Ireland. The funder had no role in the design, analysis or writing of this article. Conflict of interest: None. Authorship: F.M.Mc.A. designed the intervention; E.C.O.B. analysed data and wrote the manuscript; all authors read and approved the final manuscript; F.M.Mc.A. had primary responsibility for the final content. Ethics of buman subject participation: This study was conducted according to the guidelines laid down in the Declaration of Helsinki and all procedures involving human subjects were approved by the ethics committee at The National Maternity Hospital, Dublin, Ireland. Written informed consent was obtained from all subjects.

\section{Supplementary material}

To view supplementary material for this article, please visit https://doi.org/10.1017/S1368980017001951

\section{References}

1. McLeod ER, Campbell KJ \& Hesketh KD (2011) Nutrition knowledge: a mediator between socioeconomic position and diet quality in Australian first-time mothers. J Am Diet Assoc 111, 696-704.

2. Freisling H, Elmadfa I \& Gall I (2006) The effect of socioeconomic status on dietary intake, physical activity and body mass index in Austrian pregnant women. J Hum Nutr Diet 19, 437-445.

3. Rifas-Shiman SL, Rich-Edwards JW, Kleinman KP et al. (2009) Dietary quality during pregnancy varies by maternal characteristics in Project Viva: a US cohort. J Am Diet Assoc 109, 1004-1011.

4. Baron R, Manniën J, te Velde SJ et al. (2015) Sociodemographic inequalities across a range of health status indicators and health behaviours among pregnant women in prenatal primary care: a cross-sectional study. $B M C$ Pregnancy Childbirth 15, 261.

5. Phares TM, Morrow B, Lansky A et al. (2004) Surveillance for disparities in maternal health-related behaviors selected states, Pregnancy Risk Assessment Monitoring 
System (PRAMS), 2000-2001. MMWR Surveill Summ 53, $1-13$.

6. Smedberg J, Lupattelli A, Mårdby A-C et al. (2014) Characteristics of women who continue smoking during pregnancy: a cross-sectional study of pregnant women and new mothers in 15 European countries. BMC Pregnancy Childbirth 14, 213.

7. Larrañaga I, Santa-Marina L, Begiristain H et al. (2013) Socio-economic inequalities in health, habits and self-care during pregnancy in Spain. Matern. Child Health J 17, $1315-1324$.

8. Agyemang C, Vrijkotte TGM, Droomers M et al. (2009) The effect of neighbourhood income and deprivation on pregnancy outcomes in Amsterdam, The Netherlands. J Epidemiol Community Health 63, 755-760.

9. Metcalfe A, Lail P, Ghali WA et al. (2011) The association between neighbourhoods and adverse birth outcomes: a systematic review and meta-analysis of multi-level studies. Paediatr Perinat Epidemiol 25, 236-245.

10. Shavers VL (2007) Measurement of socioeconomic status in health disparities research. J Natl Med Assoc 99, 1013-1023.

11. Gehlert S, Sohmer D, Sacks T et al. (2008) Targeting health disparities: a model linking upstream determinants to downstream interventions. Health Aff (Millwood) 27, 339-349.

12. Sooman A \& Macintyre S (1995) Health and perceptions of the local environment in socially contrasting neighbourhoods in Glasgow. Health Place 1, 15-26.

13. Wiig K \& Smith C (2009) The art of grocery shopping on a food stamp budget: factors influencing the food choices of low-income women as they try to make ends meet. Public Health Nutr 12, 1726-1734.

14. McFadden A, Green JM, Williams V et al. (2014) Can food vouchers improve nutrition and reduce health inequalities in low-income mothers and young children: a multi-method evaluation of the experiences of beneficiaries and practitioners of the Healthy Start programme in England. BMC Public Health 14, 148.

15. Healthy Food for All (2016) 2006-2016: A Legacy to Address Food Poverty in Ireland. http://healthyfoodforall.com/ news/2016/03/2006-2016-a-legacy-to-address-food-poverty/ (accessed January 2017).

16. Dodd J \& Thangaratinam S (2016) Researchers' position statement on tackling obesity in pregnancy: the International Weight Management in Pregnancy (i-WIP) collaboration pleads for public health intervention. BJOG 123, 163-164.

17. Callander EJ \& McDermott R (2016) Measuring the effects of CVD interventions and studies across socioeconomic groups: a brief review. Int J Cardiol 227, 635-643.

18. Magin P, Victoire A, Zhen XM et al. (2013) Under-reporting of socioeconomic status of patients in stroke trials: adherence to CONSORT principles. Stroke 44, 2920-2922.

19. Furler J, Magin P, Pirotta M et al. (2012) Participant demographics reported in 'Table 1' of randomised controlled trials: a case of 'inverse evidence'? Int J Equity Health 11, 14.

20. Brownson R, Seiler R \& Eyler A (2010) Measuring the impact of public health policy. Prev Chronic Dis 7, A77.

21. Walsh JM, McGowan CA, Mahony R et al. (2012) Low glycaemic index diet in pregnancy to prevent macrosomia (ROLO study): randomised control trial. BMJ 345, e5605.

22. Kennelly MA, Ainscough K, Lindsay K et al. (2016) Pregnancy, exercise and nutrition research study with smart phone app support (Pears): study protocol of a randomized controlled trial. Contemp Clin Trials 46, 92-99.

23. Dodd JM, Turnbull D, McPhee AJ et al. (2014) Antenatal lifestyle advice for women who are overweight or obese: LIMIT randomised trial. BMJ 348, g1285.
24. Kernot J, Olds T, Lewis LK et al. (2013) Effectiveness of a facebook-delivered physical activity intervention for postpartum women: a randomized controlled trial protocol. BMC Public Health 13, 518.

25. McGowan CA, Walsh JM, Byrne J et al. (2013) The influence of a low glycemic index dietary intervention on maternal dietary intake, glycemic index and gestational weight gain during pregnancy: a randomized controlled trial. Nutr $J$ 12, 140 .

26. Institute of Medicine \& National Research Council, Committee to Reexamine IOM Pregnancy Weight Guidelines (2009) Weight Gain During Pregnancy: Reexamining the Guidelines. Washington, DC: National Academies Press.

27. World Health Organization Regional Office for Europe (1998) Wellbeing Measures in Primary Health Care: the DepCare Project. Report on a WHO Meeting Stockholm, Sweden, 12-13 February 1998. Copenhagen: WHO Regional Office for Europe.

28. Topp CW, Østergaard SD, Søndergaard S et al. (2015) The WHO-5 well-being index: a systematic review of the literature. Psychother Psychosom 84, 167-176.

29. Haase T \& Pratschke J (2012) The 2011 Pobal HP Deprivation Index for Small Areas (SA). Introduction and Reference Tables. https://www.pobal.ie/Publications/Documents/Introduction\% 20and\%20Reference\%20Tables.pdf (accessed November 2016).

30. Huynh M, Borrell LN \& Chambers EC (2014) Maternal education and excessive gestational weight gain in New York city, 1999-2001: the effect of race/ethnicity and neighborhood socioeconomic status. Matern Child Health J 18, $138-145$.

31. Kraschnewski JL, Chuang CH, Downs DS et al. (2013) Association of prenatal physical activity and gestational weight gain: results from the first baby study. Womens Health Issues 23, e233-e238.

32. McClure CK, Catov JM, Ness R et al. (2013) Associations between gestational weight gain and BMI, abdominal adiposity, and traditional measures of cardiometabolic risk in mothers 8 y postpartum. Am J Clin Nutr 98, 1218-1225.

33. Al Mamun A, Mannan M, O'Callaghan MJ et al. (2013) Association between gestational weight gain and postpartum diabetes: evidence from a community based large cohort study. PLoS One 8, e75679.

34. Sridhar SB, Darbinian J, Ehrlich SF et al. (2014) Maternal gestational weight gain and offspring risk for childhood overweight or obesity. Am J Obstet Gynecol 211, 259.e1-e8.

35. Herring SJ, Nelson DB, Davey A et al. (2012) Determinants of excessive gestational weight gain in urban, lowincome women. Womens Health Issues 22, e439-e446.

36. Guilloty NI, Soto R, Anzalota L et al. (2015) Diet, prepregnancy BMI, and gestational weight gain in Puerto Rican women. Matern Child Health J 19, 2453-2461.

37. Restall A, Taylor RS, Thompson JMD et al. (2014) Risk factors for excessive gestational weight gain in a healthy, nulliparous cohort. J Obes 2014, 148391.

38. Poston L, Bell R, Croker $\mathrm{H}$ et al. (2015) Effect of a behavioural intervention in obese pregnant women (the UPBEAT study): a multicentre, randomised controlled trial. Lancet Diabetes Endocrinol 3, 767-777.

39. Linné Y, Dye L, Barkeling B et al. (2004) Long-term weight development in women: a 15-year follow-up of the effects of pregnancy. Obes Res 12, 1166-1178.

40. Dodd JM, Cramp C, Sui Z et al. (2014) The effects of antenatal dietary and lifestyle advice for women who are overweight or obese on maternal diet and physical activity: the LIMIT randomised trial. BMC Med 12, 161.

41. Siu J, Giskes K \& Turrell G (2011) Socio-economic differences in weight-control behaviours and barriers to weight control. Public Health Nutr 14, 1768-1778.

42. Heery E, McConnon A, Kelleher CC et al. (2013) Perspectives on weight gain and lifestyle practices during 
pregnancy among women with a history of macrosomia: a qualitative study in the Republic of Ireland. BMC Pregnancy Childbirth 13, 202.

43. Fowles ER, Stang J, Bryant M et al. (2012) Stress, depression, social support, and eating habits reduce diet quality in the first trimester in low-income women: a pilot study. I Acad Nutr Diet 112, 1619-1625.

44. Heery E, Wall PG, Kelleher CC et al. (2016) Effects of dietary restraint and weight gain attitudes on gestational weight gain. Appetite 107, 501-510.

45. World Health Organization (1986) The Ottawa Charter for Health Promotion. Geneva: WHO.

46. Garcia AL, Reardon R, McDonald M et al. (2016) Community interventions to improve cooking skills and their effects on confidence and eating behaviour. Curr Nutr Rep 5, 315-322.

47. Litt JS, Soobader M-J, Turbin MS et al. (2011) The influence of social involvement, neighborhood aesthetics, and community garden participation on fruit and vegetable consumption. Am J Public Health 101, 1466-1473.

48. Docherty A, Bugge C \& Watterson A (2012) Engagement: an indicator of difference in the perceptions of antenatal care for pregnant women from diverse socioeconomic backgrounds. Health Expect 15, 126-138.

49. INVOLVE (2012) What is public involvement in research? http://www.invo.org.uk/find-out-more/what-ispublic-involvement-in-research-2/ (accessed January 2017).
50. Moss N, Daru J, Lanz D et al. (2016) Involving pregnant women, mothers and members of the public to improve the quality of women's health research. BJOG 124, 362-265.

51. Brett J, Staniszewska S, Mockford C et al. (2014) A systematic review of the impact of patient and public involvement on service users, researchers and communities. Patient 7, 387-395.

52. Lorenc T, Petticrew M, Welch V et al. (2013) What types of interventions generate inequalities? Evidence from systematic reviews: Table 1. J Epidemiol Community Health 67, 190-193.

53. Hamza O, Colin C, O'Brien H et al. (2003) Interventions comprenant des suppléments alimentaires OLO - Oeufs Lait Oranges. Montréal, QC: Comité scientifique de la Fondation OLO.

54. Schultz DJ, Byker Shanks C \& Houghtaling B (2015) The impact of the 2009 Special Supplemental Nutrition Program for Women, Infants, and Children food package revisions on participants: a systematic review. J Acad Nutr Diet 115, 1832-1846.

55. Olds DL (2002) Prenatal and infancy home visiting by nurses: from randomized trials to community replication. Prev Sci 3, 153-172.

56. Doyle O, McGlanaghy E, Palamaro-Munsell E et al. (2014) Home based educational intervention to improve perinatal outcomes for a disadvantaged community: a randomised control trial. Eur J Obstet Gynecol Reprod Biol 180, 162-167. 